\title{
H-Mode Pedestal Scaling in DIII-D, ASDEX Upgrade and JET
}

M.N.A. Beurskens' ${ }^{1}$, T.H. Osborne ${ }^{2}$, P.A. Schneider 3 , E. Wolfrum ${ }^{3}$, L. Frassinetti ${ }^{4}$, R. Groebner $^{2}$, P. Lomas ${ }^{1}$, I Nunes ${ }^{5}$, S. Saarelma ${ }^{1}$, R. Scannell ${ }^{1}$, P.B. Snyder ${ }^{2}$, D. Zarzoso ${ }^{6}$, I. Balboa1, B. Bray², M. Brix¹, J Flanagan'1, C. Giroud1, E Giovannozzi', M Kempenaars', A. Loartes, E. de la Luna', G. Maddison'1, C.F. Maggi³, D. McDonald1, R. Pasqualotto'10, G. Saibene11, R. Sartori11, E. Solano9, M. Walsh8, L. Zabeo8, the DIII-D team, the ASDEX Upgrade team, and JET-EFDA contributors ${ }^{\S}$

JET-EFDA, Culham Science Centre, OX14 3DB, Abingdon, UK

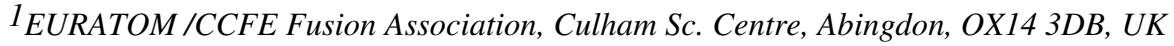

${ }^{2}$ General Atomics, PO Box 85608, San Diego, CA 92186-5608, USA

3 Association EURATOM-Max-Planck-Institut für Plasmaphysik, D-85748 Garching, Germany

${ }^{4}$ Association EURATOM-VR, Alfven Laboratory, School of Electr. Engin., KTH, Stockholm, Sweden

${ }^{5}$ Centro de Fusao Nuclear, Associacao EURATOM-IST, Lisboa, Portugal

${ }^{6}$ CEA, IRFM, F-13108 Saint Paul-lez-Durance, France

7 Associazione EURATOM -ENEA sulla Fusione, C.R. Frascati, Frascati, Italy

8 ITER Organization, CS 90 046, F-13067 Saint Paul lez Durance Cedex, France

${ }^{9}$ Associacion EURATOM-CIEMAT para Fusion, Madrid, Spain

10 Associazione EURATOM-ENEA sulla Fusione, Consorzio RFX Padova, Italy



$\S$ See Appendix of F Romanelli et al., Fusion Energy 2010 (Proc. 23rd Int Conf Daejeon, 2010) IAEA

\begin{abstract}
Multi device pedestal scaling experiments in the DIII-D, ASDEX Upgrade (AUG) and JET tokamaks are presented in order to test two plasma physics pedestal width models. The first model proposes a scaling of the pedestal width $\Delta a \propto \rho^{* 1 / 2}$ to $\rho^{*}$ based on the radial extent of the pedestal being set by the point where the linear turbulence growth rate exceeds the $E x B$ velocity. In the multi device experiment where $\rho^{*}$ at the pedestal top was varied by a factor of four while other dimensionless parameters where kept fixed, it has been observed that the temperature pedestal width in real space coordinates scales with machine size, and that therefore the gyroradius scaling suggested by the model is not supported by the experiments. This density pedestal width is not invariant with $\rho^{*}$ which after comparison with a simple neutral fuelling model may be attributed to variations in the neutral fuelling patterns. The second model, EPED1, is based on kinetic ballooning modes setting the limit of the radial extent of the pedestal region and leads to $\Delta_{\psi} \propto \beta_{p}{ }^{1 / 2}$. All three devices show a scaling of the pedestal width in normalised poloidal flux as $\Delta_{\psi} \propto \beta_{p}{ }^{1 / 2}$, as described by the kinetic ballooning model, however on JET and AUG this could not be distinguished from an interpretation where the pedestal is fixed in real space. Pedestal data from all three devices have been compared with the predictive pedestal model EPED1 and the model produces pedestal height values that match the experimental data well.
\end{abstract}




\section{Introduction}

Both the net fusion power and power production efficiency of an H-mode based tokamak reactor are expected to improve strongly with increasing pressure at the top of the H-mode pedestal. For ITER to fulfil its fusion power output goals at its design density [1], a pedestal temperature of approximately $4 \mathrm{keV}$ is required, with the exact number depending on the degree of core profiles stiffness predicted by the particular turbulent transport model. As the pedestal pressure gradient is limited by MHD-stability through combined peeling-ballooning stability $[2,3,4,5,6,7]$ the pedestal width $\Delta p_{p e d}$ determines the maximum obtainable height of the pedestal pressure, $p_{\text {ped }}$. From MHD stability analysis one can infer then that ITER would require a pedestal width of $2.5 \%$ of the minor radius to reach the required $T_{p e d}=4 \mathrm{keV}$ $[8,9]$.

A number of theoretical arguments have been made as to what might set the extent of the $E x B$ velocity shear turbulence suppression zone in the H-mode edge $[8,10,11]$. Width scalings based on this mechanism are typically derived from the point where the turbulence drive overcomes the velocity shear, i.e. where the linear turbulence growth rate exceeds the EXB velocity shearing rate. This leads to a width dependence on the normalised ion gyroradius $\left(\rho^{*}=\rho_{\text {tor }} / a \propto \sqrt{ } T_{i} / B_{T} / a\right.$, where $a$ is the minor radius in meters $)$ ranging from $\Delta_{r / a}=\Delta a \propto \rho^{* 1 / 2}$ to $\Delta_{r / a} \propto \rho^{*}$ depending on the assumed linear growth rate scaling (with $\Delta$ the outer mid-plane pedestal width in meters). Such a positive dependence of pedestal width on the ion gyro radius is a concern for ITER because $\rho^{*}$ in a large high-field tokamak like ITER will be smaller than present tokamaks; $\rho_{\text {JET }}^{*} \rho_{\text {ITER }}^{*}=2, \quad \rho_{\text {DIII- }}^{*}$ $D / \rho_{\text {ITER }}^{*}=4, \rho_{\text {AUG }}^{*} \rho_{\text {ITER }}^{*}=4$. Therefore, a significant dependence on gyro-radius could adversely affect ITER performance as the pedestal width $\Delta_{r / a}$ would be reduced compared to 
smaller devices [12]. Combined with the Peeling Ballooning stability theory this would lead to lower predictions of the pressure at the pedestal top in ITER.

Variation of $\rho^{*}$ by either a gas scan, varying the magnetic field, or changing ion isotope mass observed no or only a weak dependence of the pedestal width on gyro radius $[13,14,8$, 15, 16]. However such experiments carried out on a single device are hampered by the limited range of $\rho^{*}$ that can be covered without a mixing of the $\rho^{*}$ variation with other effects. A high priority is to conduct multi machine $\rho^{*}$ similarity experiments where $\rho^{*}$ is varied over a wider range than is possible within a single device while other dimensionless parameters such as the normalised pedestal poloidal pressure $\beta_{p, p e d}$ and pedestal collisionality $\nu_{\text {ped }}^{*}$ are kept constant $\left(\beta_{p, p e d} \propto p /<B_{p}>^{2}\right.$, where $B_{p}=u_{0} I_{p} / c_{p}$ is the averaged poloidal magnetic field (with $c_{p}$ is the plasma circumference), and $v^{*} \propto n_{e} / T_{e}{ }^{2}$ ). Experiments in which all the dimensionless quantities were matched at the top of the pedestal on two different tokamaks indicate that the electron temperature pedestal width scales with machine size minor radius as $\Delta T_{e} / a \approx 3 \%[17,18,19,20]$. However there can be differences in the behaviour of the density pedestal width, which plays an equally important role in setting the pedestal pressure height and gradient.

A simple model that incorporates the role of neutral particles on the pedestal structure is given by [22]. This model describes the density pedestal width $\Delta n_{p e d}$ as a function of the neutral mean free path $\lambda_{n-\text { free }}$ into the plasma leading to the simple relation $\Delta n_{\text {ped }} \propto \lambda_{n \text {-free }} \propto$ $1 / n_{\text {ped. }}$. Experiments in support of this model have been presented in the literature, e.g. [22,23], whereas other reports show no variation of $\Delta n_{p e d}$ with $1 / \mathrm{n}_{\text {ped }}$, e.g. [24, 25, 26, 27].

A new theoretical model, EPED1, [28] proposes that, after the drift wave turbulence is suppressed by $E x B$ velocity, the onset of short wavelength kinetic ballooning mode turbulence constrains the pedestal to a critical normalized pressure gradient, $\alpha_{C}$. Since $\alpha \propto$ 
$\beta_{p, p e d} / \Delta_{\psi}$, this gives a relation between the pedestal width $\Delta_{\psi}$ (now in normalised flux rather than in real space co-ordinates), approximately $\Delta_{\psi} \propto \beta_{p, p e d}^{1 / 2}$ once magnetic shear dependence is accounted for. This constraint is approximately consistent with earlier empirical scaling of the width as $\Delta \propto \beta_{p, p e d}^{0.4}$ [13]. A further relation between the width and height is provided by the peeling-ballooning mode instability onset condition for the ELM. These two constraints lead to a single operating point for the width and height in EPED1. Inside this pedestal model the density and temperature pedestal widths are assumed to be the same and are defined as $\left(\Delta n_{e}+\Delta T_{e}\right) / 2=\Delta n_{e}=\Delta T_{e}$. EPED1 has been used successfully to predict pedestal conditions in DIII-D and other tokamaks [28, 29]. Numerous experimental studies on the relation $\Delta_{\psi} \propto \beta_{p}{ }^{1 / 2}$ have been presented in the literature, e.g. MAST, JT-60U, Alcator C-Mod, AUG [ 15, 16, 28, 30]. All observations are consistent with $\Delta_{\psi} \propto \beta_{p}{ }^{1 / 2}$.

Inter machine comparisons are complicated by variation in device conditions. Even when the magnetic configuration is matched between devices, conditions such as pumping efficiency or proximity of the plasma to the first wall can differ largely and can affect parameters such as the background neutral pressure and recycling rates. In addition, variations in the choice of wall material e.g. carbon composite or tungsten tiles, can affect the background impurity concentration, which subsequently can influence the edge radiation pattern or lead to changes in available plasma operational space. Both affect pedestal conditions and make identity experiments a challenging exercise. Variation in e.g. background impurity concentration and its role on the pedestal formation and stability are important topics of research in future experiments in JET with the W/Be ITER-Like-Wall (ILW) and in light of the planned full metal W/Be wall in ITER.

The DIII-D and JET experiments presented in this paper are conducted with a carbon composite wall, whereas the AUG experiments were conducted in a full W-wall. The experience with the full $\mathrm{W}$-wall in AUG has shown that the achievable plasma performance 
and obtainable pedestal pressure is the same in comparison with AUG with the C-wall. However it was also found that the accessible operational space is limited to higher plasma density in AUG with the $\mathrm{W}$-wall [21]. This is bound by $\mathrm{W}$-accumulation in the core in plasmas with low plasmas density and in plasmas without central ECRH heating, and is not thought to be affected by pedestal physics. Nevertheless, as a consequence of the accessible parameter space in AUG, it has been difficult to obtain plasmas with a good dimensionless match between all three devices.

In this paper experiments are presented to test the dimensionless models for pedestal width scaling $\Delta_{\mathrm{r} / \mathrm{a}} \propto \rho^{*}$ and $\Delta_{\psi} \propto \beta_{p}{ }^{1 / 2}$ for the three devices DIII-D, AUG and JET. Advances in pedestal diagnostics, especially on JET with the new High resolution Thomson scattering system [31], have enabled detailed pedestal structure studies. This multi device experiment enables a $\rho^{*}$-pedestal width scaling study over a wider range than previously has been possible. A variation in $\rho^{*}$ by over a factor of four between JET and DIII-D has been achieved including a dimensionless match. It was also verified that atomic physics does not alter the outcome of the dimensionless study, as at the dimensionless match point $\Delta_{\mathrm{r} / \mathrm{a}, \mathrm{DIIID}}=\Delta_{\mathrm{r} / \mathrm{a}, \mathrm{JET}}$. The test of $\Delta_{\psi} \propto \beta_{p}{ }^{1 / 2}$, the relation used in EPED1, is carried out in separate experiments for each of the devices. In this paper Section II presents the experimental conditions and the specific high resolution pedestal diagnostics used in this study. In section III the dimensionless scaling experiment is described. In section IV it is shown that machine size scaling of the temperature pedestal width is valid for a variation in $\rho^{*}$ by a factor of four and thus $\Delta / \mathrm{a} \neq \rho^{*}$. Section $\mathrm{V}$ then follows to show that the experiments presented here are not inconsistent with $\Delta_{\psi} \propto \beta_{p}{ }^{1 / 2}$, the assumed width dependence in EPED1. This section also shows the pedestal height predictive capability of EPED1 for a subset of the experimental data in this paper. The paper finishes with conclusions and recommendations in section VI. 


\section{Experimental methods and analysis}

DIII-D, AUG and JET are similarly shaped tokamaks with ITER-like geometries. The three devices have similar poloidal divertor configurations with JET being a factor of 1.8 larger in linear dimension than DIII-D and AUG: AUG $\left[\mathrm{R}=1.65 \mathrm{~m}, \mathrm{a}=0.5 \mathrm{~m}, \mathrm{~V}_{\text {plasma }}=13 \mathrm{~m}^{3}\right]$, DIII-D $\left[\mathrm{R}=1.67 \mathrm{~m}, \mathrm{a}=0.54 \mathrm{~m}, \mathrm{~V}_{\text {plasma }}=16 \mathrm{~m}^{3}\right]$, JET $\left[\mathrm{R}=2.95 \mathrm{~m}, \mathrm{a}=0.95 \mathrm{~m}, \mathrm{~V}_{\text {plasma }}=80 \mathrm{~m}^{3}\right]$.

All three tokamaks are well equipped with high-spatial-resolution electron pedestal diagnostics. The DIII-D Thomson scattering system measures $\mathrm{T}_{\mathrm{e}}$ and $\mathrm{n}_{\mathrm{e}}$ along a vertical chord. The spatial coverage of the pedestal is improved by applying small vertical plasma movements. AUG combines profile measurements from three diagnostic systems: the vertical outboard Thomson scattering system in combination with small radial plasma movements gives the reference $T_{e}$ and $n_{e}$ profiles to which the electron-cyclotron emission (ECE) $T_{e}$ and lithium-beam $n_{e}$ profile are aligned with a manual procedure. Finally the JET HighResolution Thomson Scattering (HRTS) [31] measures $T_{e}$ and $n_{e}$ across a horizontal chord close the magnetic axis of the plasmas presented here. To increase the coverage of the JET measurement points in the pedestal, again small radial sweeps of the plasma by one scattering-volume width are applied. For all three devices, data are accumulated from the last 20\%-30\% of the ELM cycle for many ELMs, e.g. [19, 32]. A composite profile in normalized-flux space is then built from the many ELM cycles by mapping the profile at a specific time-point onto the flux surface geometry of the MHD equilibrium. Figure 1 shows example profiles for the three devices.

For JET and DIII-D, the pedestal parameters are determined by fitting to a modified hyperbolic tangent function (mtanh) [23] in the edge region (typically from normalized poloidal flux $=0.7$ and outwards). For AUG, the mtanh approach leads to a relatively large data scatter. For this reason the AUG pedestal profiles are fitted with a piecewise linear fit 
which on average produces similar results to the mtanh fits but with a reduced scatter [33]. Figure 1 shows example fits to the experimental profiles.

The DIII-D and AUG pedestal diagnostics offer sufficient spatial resolution ( 1\% of $a$ ) so that the instrument kernel does not significantly affect the obtained width from the pedestal fits. The spatial resolution of the JET HRTS system has been improved from July 2009 onwards from an instrument kernel with a Full-Width Half-Maximum $F W H M_{\text {kernel }} \sim 22 \mathrm{~mm}$ down to $11 \mathrm{~mm}$ as is shown in Figure 2 for two similar high triangularity JET plasmas $\mathrm{Ip}=2.5 \mathrm{MA}, \mathrm{Bt}=2.7 \mathrm{~T}, \delta=0.42, \mathrm{PNBI}=15 \mathrm{MW}$ and low gas fuelling. In this paper data from both periods are used and especially for the older data a deconvolution of the JET pedestal profiles from the instrument kernel is required [34]. The applied deconvolution procedure leads to consistent results between the two sets of data in Figure 2. The deconvolution from the $22 \mathrm{~mm}$ wide instrument kernel has a significant effect on both the derived temperature pedestal width and position, demonstrating that this procedure is essential in determining the $T_{e}$ profile structure. In a previous paper, [35], the deconvolution procedure underestimated both the solid angle of view and the effect of the angle of the field lines with respect to the laser path. As a result the width of the instrument kernel was underestimated. In addition, it has since been discovered that the data used in [35] do not have accurate calibration factors for the electron temperature in the pedestal foot leading to an underestimation of $T_{e}$ there. Unfortunately the calibration of this data cannot be recovered. This only affects plasmas with a low triangularity because of the relative position of the plasma edge with the HRTS line of sight in these plasmas. These deficiencies have been corrected in the present paper, and only data obtained in high triangularity plasma have been used for JET. In the determination of the JET pedestal width in [35] in turned out that both instrumental effects cancel out, and the qualitative conclusions in [35] are still valid and are in agreement with the results presented here. 
The pedestal structure and its stability are set by both the electron and ion temperature and density. In this paper however, the pedestal structure is obtained from electron kinetic measurements only and it is assumed that the ion pedestal structure is the same as for the electrons. The authors realise the potential shortcoming of this assumption, and a separate $T_{i}$ study is the topic of future work. As the ion pedestal temperature $T_{i, p e d}, T_{i}$ from Charge Exchange Recombination Spectroscopy (CXRS) for all three devices is taken at the location of the $T_{e}$ pedestal top. For this study the total pedestal pressure used in the $\beta_{p, p e d}$ calculation for DIII-D and JET is $p_{p e d}=\left[n_{i, p e d} X T_{i, p e d}+n_{e, p e d} X T_{e, p e d}\right]$, where $\mathrm{n}_{\mathrm{i}, \mathrm{ped}}=\mathrm{n}_{\mathrm{e}, \mathrm{ped}}\left(7-\mathrm{Z}_{\mathrm{eff}, \mathrm{ped}}\right) / 6$, assuming the effective ion charge $Z_{\text {eff,ped }}=1.5$ and Carbon as the main impurity. For AUG with the tungsten wall material the dilution effect is reduced and $\mathrm{p}_{\mathrm{ped}}=\left[n_{\text {e,ped }} X T_{i, p e d}+\right.$ $\left.n_{e, p e d} X T_{e, p e d}\right]$ is assumed.

\section{III.Dimensionless $\rho^{*}$ scaling experiment.}

In the dimensionless scan, the normalised gyroradius $\rho^{*}$, normalised pressure $\beta_{p \text {,ped }}$, and collisionality $v_{e}{ }^{*}$ are defined as:

$$
\begin{aligned}
& \rho^{*} \equiv 4.6 \times 10^{-3}\left(m^{1 / 2} / z\right)\left(T_{i}(k e V)\right)^{1 / 2} /\left(a B_{T}\right) \\
& \beta_{p, p e d}=p_{p e d} /\left(B_{p}^{2} / 2 \mu_{0}\right) \\
& v_{e}^{*}=q \times R^{5 / 2} \times a^{-3 / 2} \times\left(\lambda_{e, e}\right)^{-1}
\end{aligned}
$$

Where $m$ is the atomic mass, $z$ the atomic charge of the main ions and $\lambda_{e, e} \approx 2.245 \times 10^{18} T_{e}^{2}$ (eV) $/ n_{e}\left(\mathrm{~m}^{-3}\right)$, e.g. [36]. In order to achieve a dimensionless identity, all dimensionless parameters at the pedestal top as well as the safety factor $q_{95}$ are held fixed and the density, temperature, and plasma current scale with minor radius and toroidal field as $n_{p e d} \propto a^{-1 / 3} B_{T}^{4 / 3}$, $T_{p e d} \propto a^{1 / 3} B_{T}^{2 / 3}$, and $I_{p} \propto a B_{T}$, giving a $\rho^{*}$ variation as $\rho^{*} \propto a^{-5 / 6} B_{T}^{-2 / 3}$. This means that at the 
dimensionless identity point where $\rho^{*}$ is matched as well, $n_{\text {ped, DIII-D }} \approx 3 \times n_{\text {ped, JET }}, T_{\text {ped, DIII-D }} \approx$ $1.3 \times T_{p e d, J E T}, B_{T, D I I-D} \approx 2 \times B_{T, J E T}$, and $I_{p, D I I I-D} \approx 1.1 \times I_{p, J E T}$. For AUG similar ratios apply. The parameters chosen for the identity experiment are $I_{p}=1.0 M A, B_{T}=1.1 T$ for JET and $I_{p}=1.1 M A$ and $B_{T}=2.1 T$ for DIII-D. The $\rho^{*}$ scan is achieved by varying the magnetic field on JET in three steps as $B_{T}=1.1 T, 1.8 T$ and 2.7T and on DIII-D as $B_{T}=1 T, 1.4 T$ and 2.1T. The neutral beam input power was varied on JET from $P_{N B I}=4.5$ to $15 \mathrm{MW}$ and on DIII-D from 1.5 to $9 M W$. At the higher field a small amount of ion cyclotron heating of $P_{I C R H}=1 M W$ was applied on JET to avoid density peaking.

For DIII-D two plasma configurations have been used at low triangularity $\left(\delta_{l}=0.4\right.$ and $\left.\delta_{u}=0.2\right)$, and high triangularity $\left(\delta_{l}=0.4\right.$ and $\left.\delta_{u}=0.45\right)$ respectively. For JET only in the high triangularity configuration reliable HRTS measurements were obtained. Future experiments will deal with the low triangularity configuration in JET. Initial results for the AUG $\rho^{*}$ dimensionless scaling experiments have been obtained and are discussed later in this section. Figure 3 shows the poloidal cross-section for both shapes used in this study. The lower outer squareness could not be perfectly matched between the JET and DIII-D high triangularity shapes because of a difference in the divertor coil geometry between JET and DIII-D.

Figure 4 shows the variation in the main dimensionless parameters in the DIII-D/JET $\rho^{*}$ experiment. The value of $\rho^{*}$ at the pedestal top was varied by over a factor of four from $\sim 0.0022$ to $\sim 0.0093$. Through the $\rho^{*}$ variation, $\beta_{p, p e d}$ was kept within a factor of two, while $v_{e}{ }^{*}$, which is highly sensitive to $T_{e}, n_{e}$ and $q_{95}$, was matched only to within a factor of three. The $q_{95}$ disagreement was caused by the shape mismatch resulting in about a $10 \%$ variation. In addition the ratio $T_{i} / T_{e}$ was well matched at the identity point for the two devices at $T_{i} / T_{e} \approx 1.1$ but increased steadily from $T_{i} / T_{e} \approx 1.0$ at the lowest to $T_{i} / T_{e} \approx 1.35$ at the highest $\rho^{*}$. 


\section{Test of the $\rho^{*}$ pedestal width scaling and the role of atomic physics.}

The scaling of the temperature and density pedestal widths with $\rho^{*}$ are studied in this section. First examples are given of $T_{e}$ and $n_{e}$ profiles for two plasmas with a dimensionless match in Figure 5 (a) and (b) and for two plasmas at either end of the $\rho^{*}$-scan in Figure 5 (c) and (d). The profiles are normalised to the pedestal top temperature and density so that that their shape and relative position can be easily compared. The Temperature profiles are aligned assuming the radius of the mid-plane separatrix as $R_{s e p}=\operatorname{Pos}_{T e}+1 / 2 \Delta_{T e}$, with $\operatorname{Pos}_{T e}$ the position of the steepest $\mathrm{T}_{\mathrm{e}}$ gradient and $\Delta_{T e}$ the mid-plane temperature pedestal width. This procedure is required as for both JET and DIII-D the magnetic equilibrium reconstruction does not offer the required accuracy required to locate the relative pedestal positions. For all four profiles the temperature pedestal shape and width is invariant with $\rho^{*}$. However the density pedestal width and especially the position vary and do not show a consistent trend with $\rho^{*}$.

The electron temperature and density pedestal widths from the mtanh fits to the pedestal profiles for all plasmas used in this study are given as a function of $\rho^{*}$ in Figure 6(a) and (b) . Again the temperature pedestal width is invariant with $\rho^{*}$ and follows a machine size scaling $\Delta T_{e}^{J E T} / a=\Delta T_{e}^{D I I I-D} / a=2.5 \% \pm 0.5 \%$ while $\rho^{*}$ is varied by over a factor of four across JET and DIII-D. Therefore a strong positive variation of the temperature pedestal width with $\rho^{*}$ as suggested by modelling is excluded by this data. However the density pedestal width shows some variation with $\rho^{*}$ and yields $\Delta n_{e}{ }^{J E T} / a<\Delta n_{e}{ }^{D I I-D} / a$, as is seen in Figure 6(b). It has also been observed that the relative positions of the density and temperature profiles change and the density profile moves inwards with respect to the temperature profiles as $\rho^{*}$ increases; Figure 6(c) shows that relative mid-pedestal positions $\left(P o s_{T e}-P o s_{n e}\right)$, as obtained from the mtanh fits, increases with $\rho^{*}$. No dimensionless physics model describes a relative pedestal 
position variation as observed here. The combined weak variation of the density pedestal width and the relative variation in the $T_{e}$ and $n_{e}$ pedestal position suggest a possible role of atomic physics in setting the density pedestal structure. In the neutral penetration model [22, $37,38]$ the density pedestal width is defined as $\left|R_{t o p . n e^{-}} R_{\text {sep, }}\right|$, as given in Figure (4d), where the top of the density pedestal $R_{\text {top,ne }}=\operatorname{Pos}_{n e^{-}}-1 / 2 \Delta n_{e}$ and the foot of the temperature pedestal $R_{s e p, T e}=R_{\text {sep }}$, as defined earlier. For the DIII-D data this parameter shows a positive trend with $\rho^{*}$. Also this parameter is not conserved at the dimensionless match point and we find $I R_{t o p . n e^{-}}$ $\left.R_{\text {sep, } T e}\right|^{D I I I-D} / a<\left|R_{\text {top.ne }}-R_{\text {sep }, T e}\right|^{J E T} / a$ at $\rho_{\text {match }}^{*} \approx 0.004$.

The neutral penetration model in $[22,37]$ predicts a pedestal density dependence of the density pedestal structure as $\left|R_{\text {top.ne }}-R_{\text {sep }}\right| \propto 1 /\left(n_{\text {ped }} E^{*}\right)$, where $E^{*}$ is the poloidally averaged flux expansion weighted with the magnitude of the neutral particle source. Figure 7(a) shows that the density pedestal width itself does not show a strong variation with $1 / n_{e, p e d}$. The weak positive trend in $\Delta n_{e}$ with $\rho^{*}$ can therefore not be explained with this simple model. However Figure 7(b) shows that $\operatorname{Pos}_{\mathrm{Te}}-\mathrm{Pos}_{\mathrm{ne}}$ is positively correlated to $1 / n_{e, p e d}$, and leads to the $\left|R_{t o p . n e}-R_{\text {sep,Te }}\right|$ variation as shown in Figure $7(\mathrm{c})$. For DIII-D the width $\left|R_{\text {top.ne }}-R_{\text {sep, } T e}\right|^{\text {DIII-D }}$ closely follows a $1 / n_{e, p e d}$ trend, whereas for JET $\left|R_{t o p . n e^{-}} R_{\text {sep, } T e}\right|^{J E T}$ does not. Also the neutral penetration model is independent of the machine size and for a given $\mathrm{n}_{\mathrm{e} \text {,ped }}$ one would expect $\left|R_{\text {top.ne }}-R_{\text {sep, } T e}\right|^{J E T}=\left|R_{\text {top.ne }}-R_{\text {sep, } T e}\right|^{D I I I-D}$. However, at fixed $1 / \mathrm{n}_{\mathrm{e}, \mathrm{ped}}=0.2 \times 10^{-19} \mathrm{~m}^{3}$ the density pedestal as defined by the neutral penetration model is $\left|R_{\text {top.ne }}-R_{\text {sep }, T e}\right|^{D I I I-D} \approx 1 / 2 \mid R_{\text {top.ne }}$ $\left.R_{\text {sep, } T e}\right|^{J E T}$. Within the model this would imply that $E^{* \mathrm{JET}} \approx 1 / 2 E^{* \mathrm{DIII}-\mathrm{D}}$. No direct measurements of the poloidally distributed neutral density are available on either of the devices, whereas such measurements would be essential in order to confirm this difference. The comparison $E^{* \mathrm{JET}} \approx 1 / 2 E^{* \mathrm{DIII}-\mathrm{D}}$ would imply that the fuelling pattern is more poloidally symmetric on JET compared to DIII-D. In conclusion The DIII-D/JET $\rho^{*}$ pedestal scaling experiments provide corroborating evidence that the temperature pedestal width does not scale with $\rho^{*}$ whereas the 
density pedestal width shows an altogether more complex dynamics for which an explanation may be found through the role of neutral penetration.

The AUG experiment on the $\rho^{*}$ scaling of the pedestal width is ongoing and no data can yet be presented here. So far, no dimensionless match has been obtained between AUG and the other two devises. The AUG metal wall introduces boundary conditions under which simultaneous operation at low plasma density and thermal $\beta_{\mathrm{N}} \sim 1.5$, as used in the experiment in the other devices, has not been possible without increased density peaking and/or impurity accumulation. The accessible parameter range on JET with the carbon wall would have allowed operation at elevated plasma density and increased $\beta_{\mathrm{N}} \sim 2$ in order to obtain the match in dimensionless parameters with AUG while maintaining good type I ELMy H-mode conditions. However with the current Be/W JET wall material, the accessible parameter range needs to be investigated first before further dimensionless scaling experiments can be conducted.

For AUG an attempt has been made to get a first indication of the $\rho^{*}$-dependence of the pedestal width. The dataset used here is well described in [30], reanalysed with the linear fit method [33], and contains a power scan at $q_{95} \sim 4.8$ and $\delta=0.23$. It is important to note that this experiment describes a power scan from $P_{N E T}=6.5-13.5 \mathrm{MW}$ and is not a dimensionless experiment as the collisionality varies as $0.9>v_{\mathrm{e}} *>0.05$ and is correlated to $0.3<\beta_{\text {p,ped }}<0.7$ through $\beta \sim n \times T$ vs $v \sim n_{e} / T_{e}^{2}$ (at relatively fixed $n_{e}=6 \times 10^{19} \mathrm{~m}^{-3}$ in the scan). Figure 8 shows the variation of the temperature and density pedestal width vs $\rho^{*}$ in this experiment. No variation of both the temperature and density pedestal width is observed with $\rho^{*}$. Note however that obtaining a dimensionless scaling experiment based on the existing JET and DIII-D data set is not possible as AUG does not have access to a low enough density. Future experiments will address this issue by creating JET and DIII-D comparison pulses at higher density. 


\section{Predictive pedestal model EPED1 and $\beta_{p}$ scaling of the pedestal width}

EPED1 is a predictive pedestal model $[28,29]$ in which the pressure width (assuming $\Delta p_{e}=$ $\left.\left(\Delta n_{e}+\Delta T_{e}\right) / 2\right)$ is constrained by kinetic ballooning modes, whose onset is only weakly affected by velocity shear, giving a scaling $\Delta_{\psi} \sim \sqrt{ } \beta_{p, p e d}$ EPED1 then couples this width scaling with a full (width dependent) calculation of the peeling-ballooning mode stability using the ELITE code [9] to determine the pressure pedestal width and height self consistently. The explicit form for the simplified KBM relation in EPED1 is given in normalized poloidal flux space as $\Delta_{\psi}=\left(\Delta n_{e}+\Delta T_{e}\right) / 2=0.076 \sqrt{ } \beta_{p, p e d}$. In the updated version of the model, EPED1.6 [39], the KBM relation is calculated directly for each case, and it is found that for AUG, JET and DIII-D $\Delta_{\psi}=G\left(v^{*}, \varepsilon\right) \sqrt{ } \beta_{p, p e d}$, where $G$ is a slowly varying function with typical values in the range of 0.07-0.1.

In this section three experiments are described in which the normalised poloidal pressure $\beta_{p, p e d}$ was varied in order to test the dependence $\Delta_{\psi} \propto \sqrt{ } \beta_{p \text {,ped }}$. By varying the input power alone in Type I ELMy H-mode discharges at fixed configuration, $q_{95}, I_{p}$, and $B_{T}$ the value of $\beta_{p}$ can be varied only by a factor of two. For this reason a dedicated experiment was conducted at DIII-D to test the EPED1 model where $\beta_{p \text {,ped }}$ was varied by a factor of seven from $\beta_{p, p e d}=0.2$ to 1.4 through varying $\left(B_{T}, I_{p}, B_{T} / I_{p}, P_{n e t}\right.$, and $\left.\delta\right)[27,28]$. For AUG and JET such a wide scan has not been performed and the experiment here describe power scans in a fixed shape taken from $[30,33]$ for AUG described in the previous section with $\beta_{p \text {,ped }}=0.3$ to 0.6 , and for JET $\beta_{p \text {,ped }}=0.2$ to 0.4 . The JET scan is conducted in the same plasma configuration as the $\rho^{*}$ scan at $I_{p}=1.7 M A, B_{t}=1.8 T, q_{95}=3.6$. Note that these are separate experiments for each of the three devices and do not constitute a dimensionless scan. 
Figure 9(a) shows that for all three data sets the core versus the pedestal pressure follow a fixed ratio of $\beta_{p \text {,ped }} / \beta_{p, \text { total }} \approx 35 \%$. This is true even for the large range of $\beta_{p \text {,ped }}$ cover in DIIID. This tight link between pedestal and total $\beta_{p}$ on all three devices leads to a fixed relation between $\beta_{p \text {,ped }}$ and the outer midplane flux surface compression $d \psi_{N} / d r$ in the pedestal region as is shown in Figure 9(b). In addition to the equilibrium data a shaded area shows a $d \psi_{N} / d r$ $\propto \sqrt{ } \beta_{p, p e d}$ trend which represents the variation in the AUG and JET data of $d \psi_{N} / d r$ well over the relatively narrow range of $\beta_{p \text {,ped }}$ covered in these two experiments. Even over the much wider range of $\beta_{p \text {,ped }}$ covered in the DIII-D experiment the $d \psi_{N} / d r \propto \sqrt{ } \beta_{p, p e d}$ is a reasonable description of the pedestal equilibrium data in the outer midplane although the trend is broken for the lowest and highest $\beta_{p \text {,ped }}$ values.

Figure 10 shows that the $d \psi_{N} / d r \propto \sqrt{ } \beta_{p, p e d}$ trend in the equilibrium data is a concern for the interpretation of the pedestal width data in terms of the $\Delta_{\psi} \propto \sqrt{ } \beta_{p, p e d}$ model. Figure 10(a) shows that for the AUG power scan the pedestal width in the outer midplane is invariant as a function of $\sqrt{ } \beta_{p \text {,ped. }}$. Figure 10(b) however shows that when mapped to normalised flux space the AUG data are consistent with $\Delta_{\psi} \propto \sqrt{ } \beta_{p, p e d}$. The inset of Figure 10(a) shows that the AUG pedestal diagnostics are located close to the outer magnetic midplane. For this reason a direct observation of $\Delta_{\psi} \propto \sqrt{ } \beta_{p, p e d}$ in the data is not possible with the AUG diagnostics in their current location. The same is true for the JET Thomson scattering measurements which are also located in the outer magnetic midplane. In order to see a strong variation along the line of sight of diagnostic measurements, these would be best located on the inner magnetic midplane where the flux surfaces expand when $\beta_{p}$ increases. A relation $\Delta_{\psi} \propto \sqrt{ } \beta_{p, p e d}$ variation would be enhanced in such an inner midplane line of sight measurement, e.g. [40]. 
Figure 11 shows the results for all three devices together. Figure 11(a) shows that also the JET pedestal width in the outer midplane is invariant with $\beta_{p, p e d}$. The DIII-D data-set spans a much wider range of $\beta_{p, p e d}$ and the averaged pedestal width shows a somewhat positive upward trend with increasing $\sqrt{ } \beta_{p, p e d}$. However, the spread in the pedestal width data is considerable and a more extensive data base is required to affirm this trend for DIII-D. The planned upgrade of the DIII-D Thomson scattering system in preparation of the 2011 experimental campaign will provide a better signal to noise performance and future experiments at high $\beta_{p, p e d}$ (with relatively low pedestal density and high pedestal temperature) are planned to improve the database.

Figure 11(b) shows that all data of DIII-D, as well as JET and AUG are consistent with $\Delta_{\psi} \propto \sqrt{ } \beta_{p, p e d}$ and that the coefficient $G\left(\Delta_{\psi}=G\left(v^{*}, \mathcal{E}\right) \sqrt{ } \beta_{p, p e d}\right)$ are approximately in the expected range of $0.07-0.10$. However, extra vigilance has been applied in the study of the spatial resolution of the diagnostic systems of these devices, to ensure that the pedestal width is resolved by the subsequent instruments. The JET data used in Figure 11 have been taken after July 2009 when the HRTS system was upgraded from 22 to $11 \mathrm{~mm}$ spatial resolution in the pedestal region. The actual averaged pedestal width is around $2.3 \mathrm{~cm}$, i.e. well resolved with the $11 \mathrm{~mm}$ resolution. This is important, since in the case where the spatial resolution of the diagnostics would be limiting the width measurements, the same $\Delta_{\psi} \propto \sqrt{ } \beta_{p, p e d}$ trend would be observed through the flux surface mapping. For future experiments a wider range of $\beta_{p, p e d}$ needs to be covered on JET and AUG to break the $d \psi_{N} / d r \propto \sqrt{ } \beta_{p \text {,ped }}$ trend in the equilibrium mapping as is demonstrated for the lowest and highest $\beta_{p \text {,ped }}$ values in the DIII-D scan in Figure 9(b). JET is also able to measure high field side electron temperature with its ECE radiometer system in O-mode for a limited range in magnetic field [41] which will be exploited. 
The data presented in this paper are consistent with the EPED1 pedestal width assumption $\Delta_{\psi}$ $\propto \sqrt{ } \beta_{p, p e d}$. Next, the model has been run on a representative subset of the data discussed to test its predictive capability. EPED1 uses simplified equilibria and only $B_{T}, I_{P}, R, a, \kappa, \delta$, and $\beta_{N}$ as input parameters from the experiment. The pedestal temperature and thus $\beta_{p, p e d}$ is adjusted self-consistently increasing the pedestal width while keeping the global $\beta_{N}$ fixed inside the model until the pressure gradient $\alpha$ exceeds the critical pedestal pressure gradient $\alpha_{c}$ in the ELITE Peeling Ballooning stability calculations. Output of the model are the predicted pedestal pressure and the pedestal width averaged pedestal width $\Delta_{\psi}=\left(\Delta_{\psi}^{n e}\right.$ $\left.+\Delta_{\psi}^{\mathrm{Te}}\right) / 2$. Figure 12 shows the good agreement between the predicted pedestal height by EPED1 and the experimentally found pedestal height $\left(2 x p_{e, p e d}\right)$.

\section{VI conclusions}

The correct analysis of the pedestal profiles including the deconvolution with the instrument kernel has proven essential for the older JET data where $\mathrm{FWHM}_{\mathrm{kernel}} \sim 22 \mathrm{~mm}$. The JET results presented here include both low $\left(\mathrm{FWHM}_{\text {kernel }}=22 \mathrm{~mm}\right)$ and high resolution $\left(\mathrm{FWHM}_{\mathrm{kernel}}=11 \mathrm{~mm}\right)$, and the deconvolution procedure leads to consistent results between the two sets of data.

Multi device pedestal scaling experiments have been presented in order to test two plasma physics pedestal width models. The first model proposes a scaling as $\Delta_{r / a} \propto \rho^{* 1 / 2}$ to $\rho^{*}$ based on the radial extend of the pedestal being set by the point where the linear turbulence growth rate exceeds the $E x B$ velocity. The second model is based on kinetic ballooning modes setting the limit of the radial extent of the pedestal region and leads to $\Delta_{\psi} \propto \beta_{p}{ }^{1 / 2}$ (now in normalised flux rather than in real space co-ordinates) 
The first model in particular would lead to adverse pedestal width predictions for future fusion devises as larger devices would operate at lower values of $\rho^{*}$ than existing ones. It has been shown in section IV that in a dimensionless scan where $\rho^{*}$ at the pedestal top was varied by a factor of four across JET and DIII-D, the temperature pedestal width remains unvaried at $\Delta T_{e} / a=2.5 \% \pm 0.5 \%$ and scales with machine size. However, the density pedestal width shows a weak positive trend with $\rho^{*}$, which may be due to a weak positive gyro-radius scaling. Also the density pedestal position with respect to the temperature pedestal position does not remain fixed and shows a strong positive trend with $\rho^{*}$. The neutral penetration model in [37] predicts $\left|R_{\text {top.ne }}-R_{\text {sep,Te }}\right| \sim 1 /\left(n_{\text {ped }} E^{*}\right)$ and it is found that the DIII-D data are consistent with such a trend indicating a possible role of neutrals setting the pedestal structure. This trend is however not reproduced in the JET data. Due to the lack of poloidally averaged neutral particle density measurements this model cannot be validated. However, between JET and DIII-D a different poloidal distribution of the neutral fuelling is required to obtain the observed $\left|R_{\text {top.ne }}-R_{\text {sep, } T e}\right|^{J E T}=2 \times\left|R_{\text {top.ne }}-R_{\text {sep, } T e}\right|^{D I I I-D}$. For the model to fit both the JET and DIII-D data $E^{* J E T}=1 / 2 E^{* D I I I-D}$ would be needed, which implies that the JET fuelling pattern is poloidally more symmetric than on DIII-D.

The density profile dynamics appear complex and cannot be fully explained by the models addressed in this paper. A possible role of neutral fuelling may be present and needs thorough future investigation. It is clear however that the $T_{e}$ and $n_{e}$ pedestal behave differently and both their relative position variation as well as the observed weak positive density pedestal width variation with $\rho^{*}$ will affect the pedestal MHD stability. It is therefore important that future predictive pedestal models include such independent $T_{e}$ and $n_{e}$ pedestal variations to test their influence on predictions towards ITER. ITER will operate both at higher density and lower $\rho^{*}$ compared to the devices discussed here. According to the study performed here both of these would lead to the density pedestal further out compared to the 
temperature pedestal. The (de-)stabilising effect and effect on the pedestal height prediction of such a variation is under investigation.

The underlying width dependence in EPED1, $\Delta_{\psi} \propto \sqrt{ } \beta_{p \text {,ped }}$, has been tested against pedestal width measurements from three experiments on respectively DIII-D, AUG and JET. On the latter two devices it has been observed that the scaling of $\left(\Delta_{\psi}^{n e}+\Delta_{\psi}^{\mathrm{Te}}\right) / 2 \propto \sqrt{ } \beta_{p, p e d}$ can be explained in terms of a constant pedestal width measurement in radial outer midplane coordinates and a flux surface compression at increasing global $\beta_{p}$. The test of this model is complicated by the fact that both the AUG and JET pedestal diagnostics are located on the outer midplane where a scaling of $\Delta_{\psi} \propto \sqrt{ } \beta_{p \text {,ped }}$ is compensated by the flux surface compression in the diagnostic measurements. It is recommended to increase the range of $\beta_{\mathrm{p} \text {,ped }}$ covered in these devices and to conduct high field side measurements of the pedestal width. Nevertheless the AUG and JET observations are not inconsistent with the $\Delta_{\psi} \propto \sqrt{ } \beta_{p, p e d}$ scaling.

Finally the predictive capability of the EPED1 model has been tested against a subset of plasmas presented in this paper. Generally a good agreement is found between the model output and the experimental data. However the underlying kinetic ballooning model implying $\Delta_{\psi} \propto \sqrt{ } \beta_{p, p e d}$ has not been sufficiently supported by the observations on JET and AUG because of the limited range of $\beta_{p, p e d}$ covered and the diagnostic limitations discussed above. The DIII-D comparisons cover a sufficiently wide coverage to decouple the link between flux expansion and $\Delta_{\psi} \propto \sqrt{ } \beta_{p, p e d}$. However, here is it is recommended that more coverage is provided for low and high $\beta_{p, p e d}$, exploiting the planned upgrade in the DIII-D Thomson scattering system providing improved signal to noise and higher spatial resolution. Also, the EPED1 model predicts an overall pedestal width in flux space, it does not separately predict the density and temperature widths, or the displacement between the density and temperature 
profiles. In the future, it is of interest to extend models to include more detailed source and transport physics to further elucidate the full complexity of the observations.

\section{Acknowledgement:}

This work, part-funded by the European Communities under the contract of Association between EURATOM and CCFE, was carried out within the framework of the European Fusion Development Agreement. The views and opinions expressed herein do not necessarily reflect those of the European Commission. This work was also part-funded by the RCUK Energy Programme under grant EP/G003955.

\section{References}

[1] Editors of 'Progress in the ITER Physics Basis', Nucl. Fusion 47 (2007) S1-S17

[2] J. W. Connor et al, Phys. Plasmas, 5, 2687 (1998)

[3] J.R. Ferron, et al, 26th EPS Conf. on Plasma Phys. And Contr. Fusion, 23J, 1201 (1999)

[4] H. R. Wilson, et al, Phys. Plasmas, 9, 1277 (2002).

[5] P. B. Snyder, et al, Phys. Plasmas 9, 2037 (2002)

[6] G. T. A. Huysmans, Plasma Phys. Controlled Fusion 47, B165 (2005).

[7] S. Saarelma, et al, Plasma Phys. Control. Fusion 51 (2009)

[8] T.H Osborne et al, 19th IAEA Fusion Energy Conference, October 14-19, 2002, Lyon, France

[9] P.B. Snyder, et al, Nucl. Fusion 44, 320 (2004).

[10] A.E. Hubbard et al, Plasma Phys. Control. Fusion 42 A15 (2000)

[11] T. Onjun et al, Physics of plasmas V9, number 12, (2002) 5018-5030

[12] A.W. Leonard et al, Journal of Physics: Conference Series 123 (2008) 012001

[13] T.H Osborne et al, Plasma Phys. Control. Fusion 40 (1998) 845-850

[14] G. Saibene et al, Nuclear Fusion, Vol. 39, No. 9, 1999, 1133

[15] A. Kirk et al, Plasma Phys. Control. Fusion 46 (2004) A187-A194

[16] H. Urano et al, Nucl. Fusion 48 (2008)

[17] D.A. Mossessian, et al, Physics of plasmas V10, number 3, (2003) 689-698

[18] T.H. Osborne et al, 45th APS Div. of Pl. Phys Meeting, October 27-31, 2003, Albuquerque, US, [KP1.023]

[19] M.E. Fenstermacher, T.H. Osborne et al, Nucl. Fusion 45 (2005) 1493-1502 
[20] G Maddisson, Nucl. Fusion 49 (2009) 125004

[21] R. Neu et al, Phys. Scr. T138 (2009) 014038

[22] M.A. Mahdavi et al, Nucl. Fusion 42 (2002) 52-58

[23] R.J. Groebner et al, Plasma Phys. Control. Fusion 44 (2002) A265-A272

[24] I. Nunes et al, Nucl. Fusion 44 (2004) 883-891

[25] I. Nunes et al, Nucl. Fusion 45 (2005) 1550-1556

[26] J. Hughes et al., Physics of Plasmas 13, 056103 (2006)

[27] R.J. Groebner et al Nucl. Fusion 49 (2009) 085037

[28] P.B. Snyder, et al., Phys. of Plasmas 16, 056118 (2009)

[29] P.B. Snyder, et al, 22nd IAEA Fusion energy conference, October 13 - 18, Geneva, (2008)

[30] C.F. Maggi et al. Nucl. Fusion 50 (2010) 025023 (20pp)

[31] R. Pasqualotto et al 2004 Rev. Sci. Instrum. 753891

[32] M.N.A Beurskens et al, Nucl. Fusion 48 (2008) 095004

[33] P. Schneider et al, 37th EPS Conference on Plasma Physics 2010, Dublin, P2.157

[34] R Scannell et al, submitted to Rev. Sci. Instrum.

[35] M.N.A Beurskens et al, Plasma Phys. Control. Fusion 51 (2009) 124051

[36] A. Loarte et al, Plasma Phys. Control. Fusion 45 (2003) 1549-1569

[37] M.A. Mahdavi et al, Phys. of Plasmas nr10, V10, p3984 (2003)

[38] A. Kallenbach et al. / Journal of Nuclear Materials 337-339 (2005) 381-385

[39] P.B. Snyder, et al, submitted to Nucl. Fusion

[40] A. Kirk et al, Plasma Phys. Control. Fusion 51 (2009) 065016

[41] L. Barrera et al, Plasma Phys. Control. Fusion 52 (2010) 085010 


\section{Figure captions :}

Figure 1: example temperature pedestal profiles and their fits for a) DIII-D with an mtanh fit to all data points, b) AUG with a linear fit to selected data points in blue filled bullets c) JET with an mtanh fit to all data points. The instrument kernel representing the spatial resolution of each of the diagnostic systems are shown in blue and the mtanh fits deconvolved with the instrument kernel are shown in red for DIII-D and JET. For JET the instrument profile plays an important role: without deconvolution the fit to the JET data indicates a width of $w_{\text {direct }}=$ $4.28 \pm 0.25 \mathrm{~cm}$, whereas after deconvolution the actual pedestal width is $w_{\text {deconvolv }}=2.26 \pm$ $0.21 \mathrm{~cm}$. For DIII-D the effect of the deconvolution is a 5-10\% reduction of the temperature pedestal width whereas for AUG no additional correction for the instrument kernel is applied.

Figure 2: Pedestal fits for two similar JET plasmas, Ip=2.5MA, Bt=2.7T, $\delta=0.42$, $P N B I=15 M W$ and low gas fuelling. Figure a) and b) are respectively the profiles of temperature and density before the spatial resolution of the HRTS system was improved and Figure c) and d) after the improvement. The instrument kernels (with arbitrary amplitude) are drawn in blue and green shaded areas for the old and new spatial resolutions respectively. The fits with deconvolution (drawn blue line) and without deconvolution (dashed black line) are shown.

Figure 3: (a) Low and (b) high triangularity configurations used in the $\rho^{*}$ dimensionless scaling experiment.

Figure 4: Variations in pedestal dimensionless parameters in the $\rho^{*}$ scan. The grey vertical bar indicates the region where a dimensionless $\rho^{*}$ match was achieved between JET and 
DIII-D in a) Normalized poloidal pressure, $\left.\beta_{p, p e d}, b\right)$ electron collisionality, $\left.{ }^{*}, c\right)$ electron to ion temperature ratio, d) safety factor, $q_{95}$.

Figure 5: Example temperature and density profiles normalised to the pedestal top values for the (a) and (b) the $\rho^{*}$-match and (c) and (d) for two extremes in the $\rho^{*}$ scan. The chosen $\rho^{*-}$ data point are indicated in Figure 6.

Figure 6: Pedestal structure parameters for JET and DIII-D as a function of $\rho^{*}:$ (a) Electron temperature pedestal width from the mtanh fit in the outer midplane normalised to the minor radius, (b) electron density width from the mtanh fit normalised to the minor radius, (c) relative position of the middle of temperature pedestal versus the middle of the density pedestal Pos Te $^{-P o s}$ ne and (d) Position of the density pedestal top minus the position of the temperature pedestal foot normalised to the minor radius $\left|R_{\text {top.ne }}-R_{\text {sep,Te }}\right| / a$. The examples used in Figure 5 are indicated in the figure.

Figure 7: a) Density pedestal width, b) relative position of the temperature pedestal and density pedestal $\operatorname{Pos}_{T e}-P_{\text {os }}$ c) Position of the density pedestal top minus the position of the temperature pedestal foot, $\left|R_{\text {top.ne- }} R_{\text {sep,Te }}\right|$, versus $1 / n_{e, p e d}$ for JET and DIII-D.

Figure 8: AUG power scan. a) Temperature and b) density pedestal width in the outer mid plane versus $\rho^{*}$.

Figure 9: a) pedestal versus total normalised poloidal pressure in the AUG, DIII-D and JET $\beta_{p, p e d}$-scans. the line indicates a ratio of $\left.\beta_{p, p e d}=0.35 \times \beta_{p, t o t a l} b\right)\left(d \psi_{N} / d r\right) \times a$ versus $\beta_{p, p e d}$ for all three devices. The blue shaded area indicates a $\sqrt{ } \beta_{p, p e d}$ trend. 
Figure 10: (a) AUG averaged width of the temperature and density pedestal in outer midplane radial coordinates versus $\sqrt{ } \beta_{p, p e d}$ (b) AUG averaged width in normalised poloidal flux versus $\sqrt{ } \beta_{p, p e d}$; dashed line is a fit at $0.1 \times \sqrt{ } \beta_{p, p e d}$.

Figure 11: averaged $T_{e}$ and $n_{e}$ pedestal width for AUG, JET and DIII-D in (a) outer midplane coordinates and in (b) normalised poloidal flux versus $\sqrt{ } \beta_{p, p e d}$. For illustration the horizontal lines in Figure (a) indicated the averaged width for each of the devices and the dashed lines in Figure (b) indicate the $0.076 \times \sqrt{ } \beta_{p, p e d}, 0.087 \times \sqrt{ } \beta_{p, p e d}$ and $0.1 \times \sqrt{ } \beta_{p, p e d}$ data trends.

Figure 12: Comparison of EPED1 prediction with measured pedestal pressure ( $\left.2 x_{e, p e d}\right)$ 
Temperature
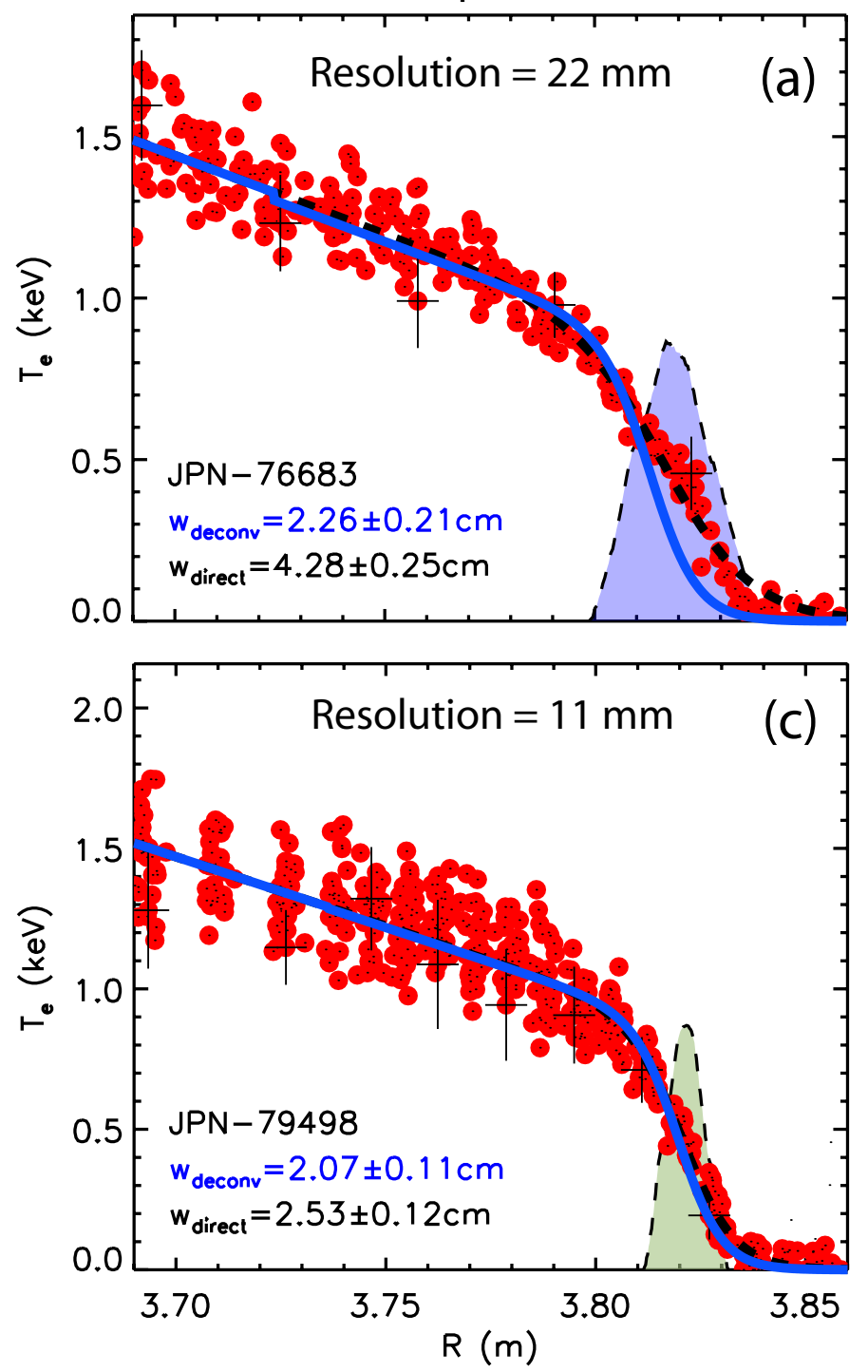

Density
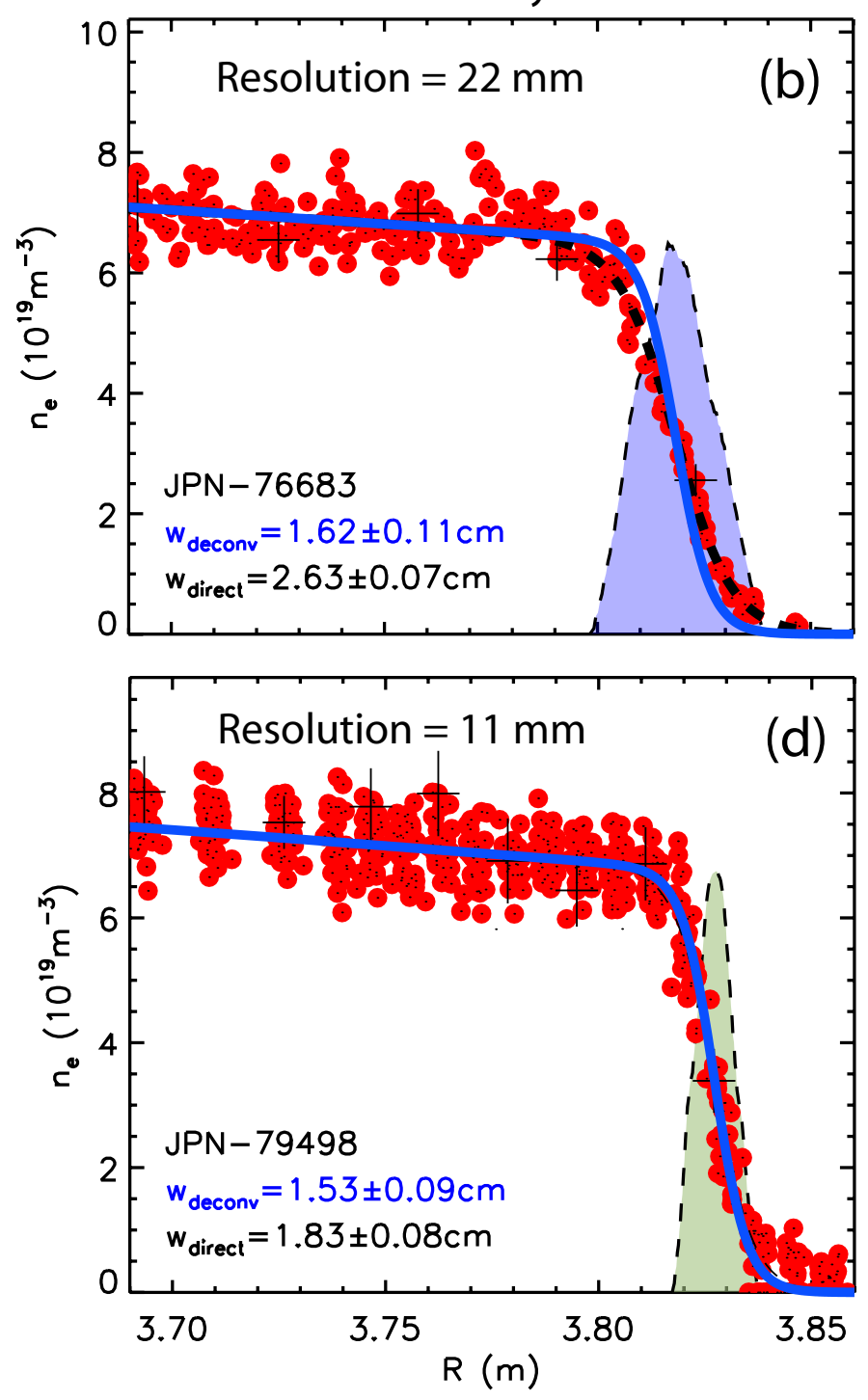

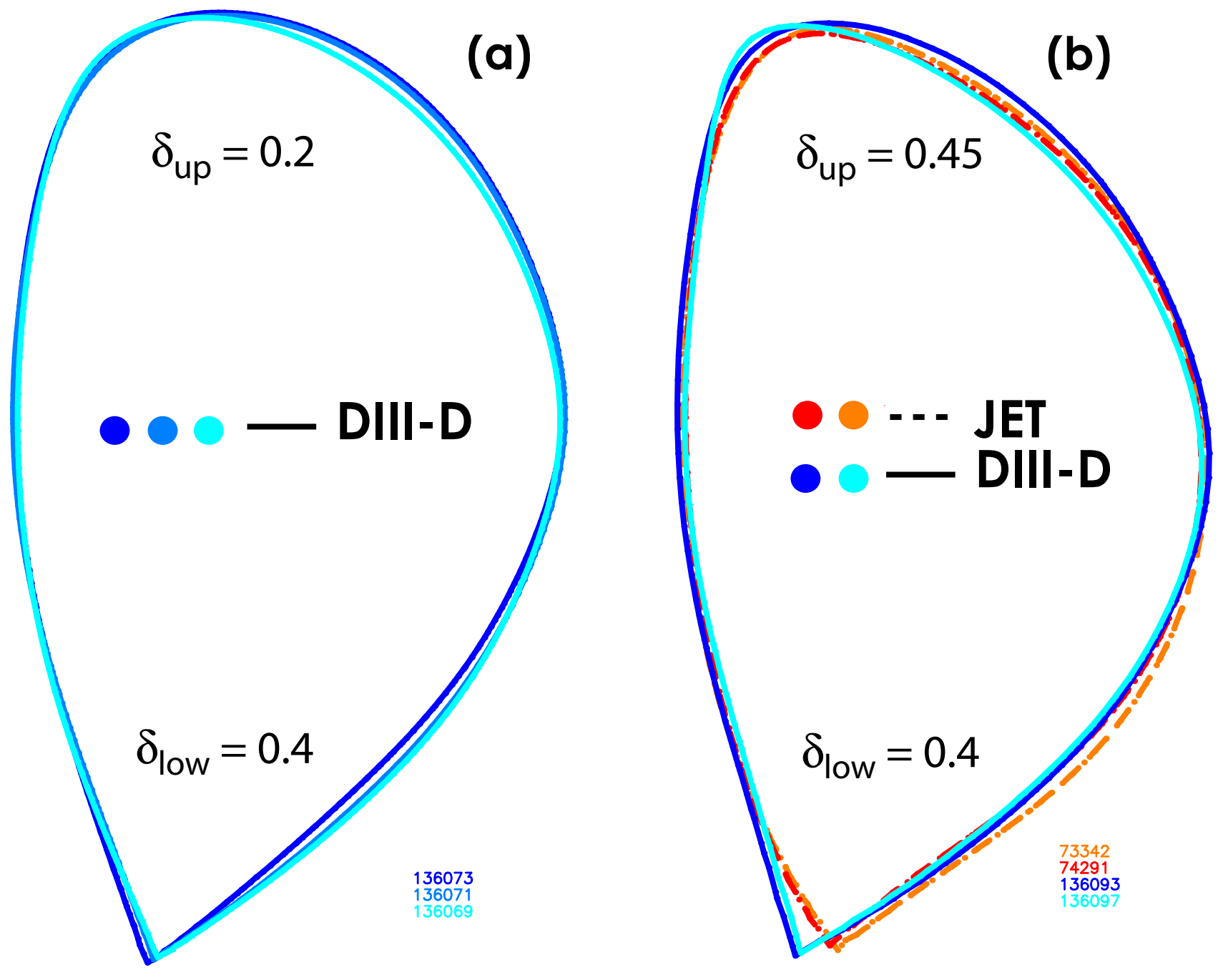

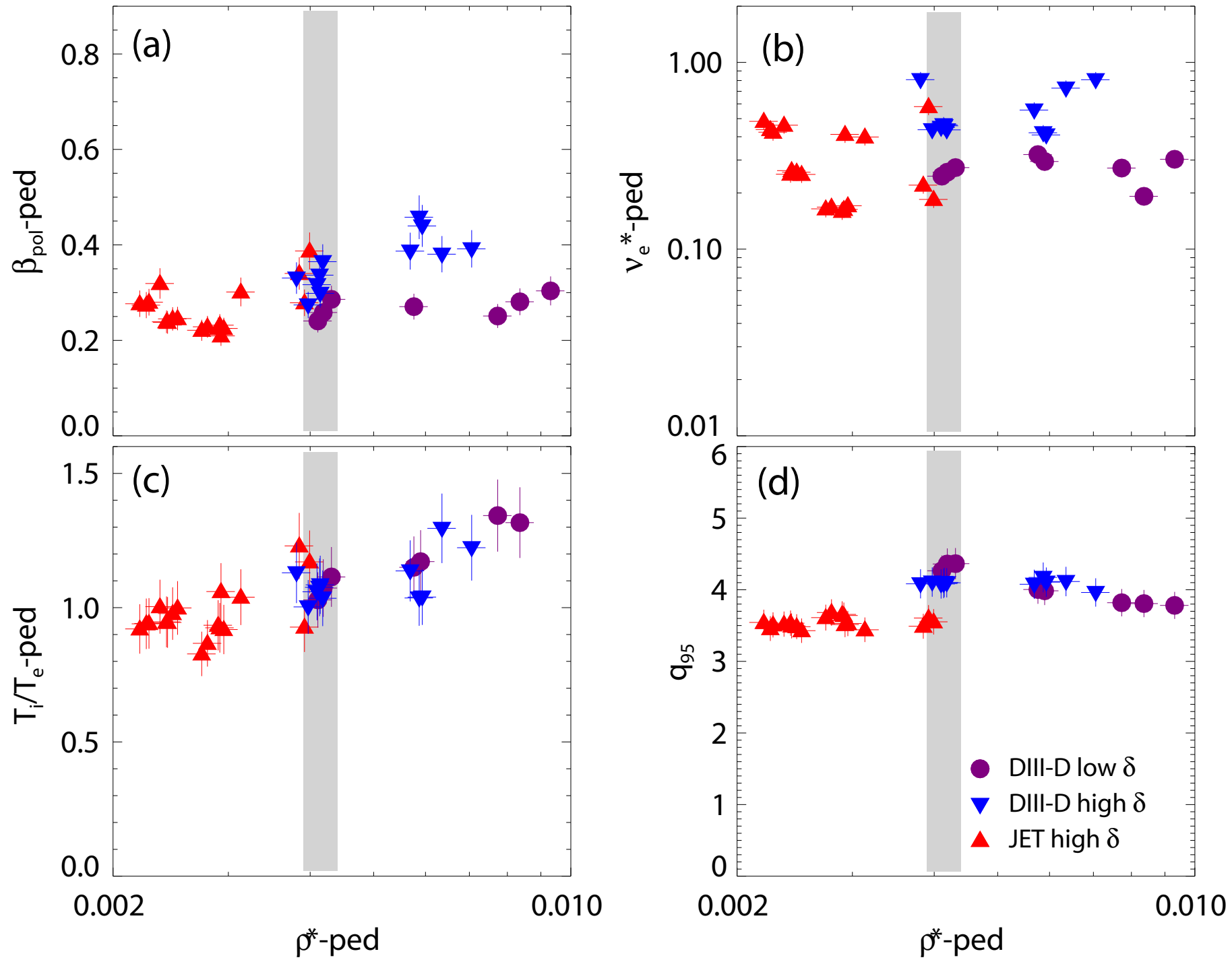



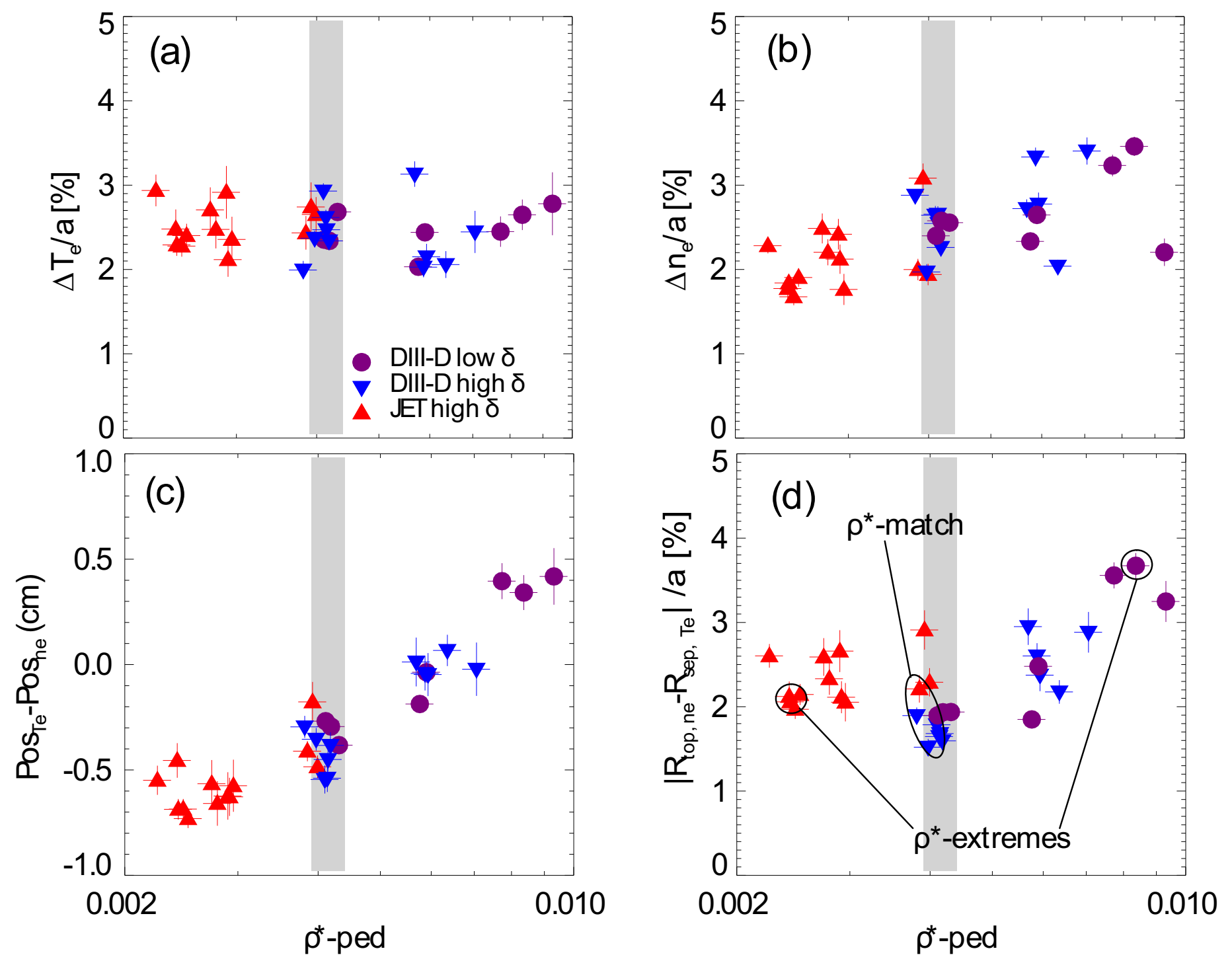

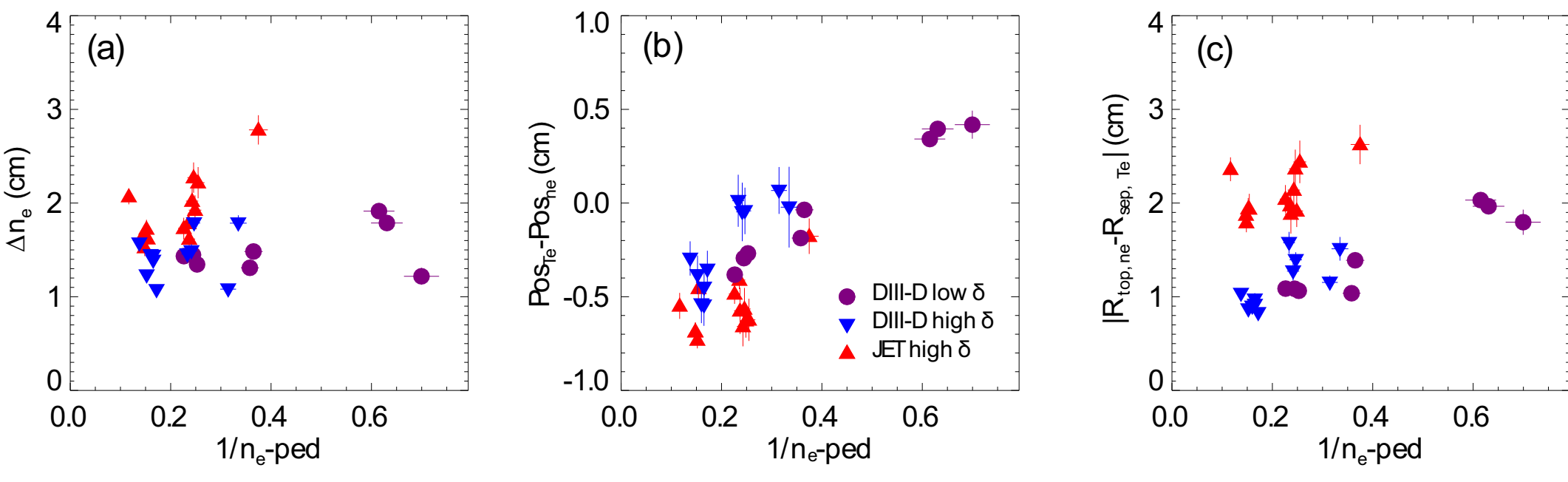

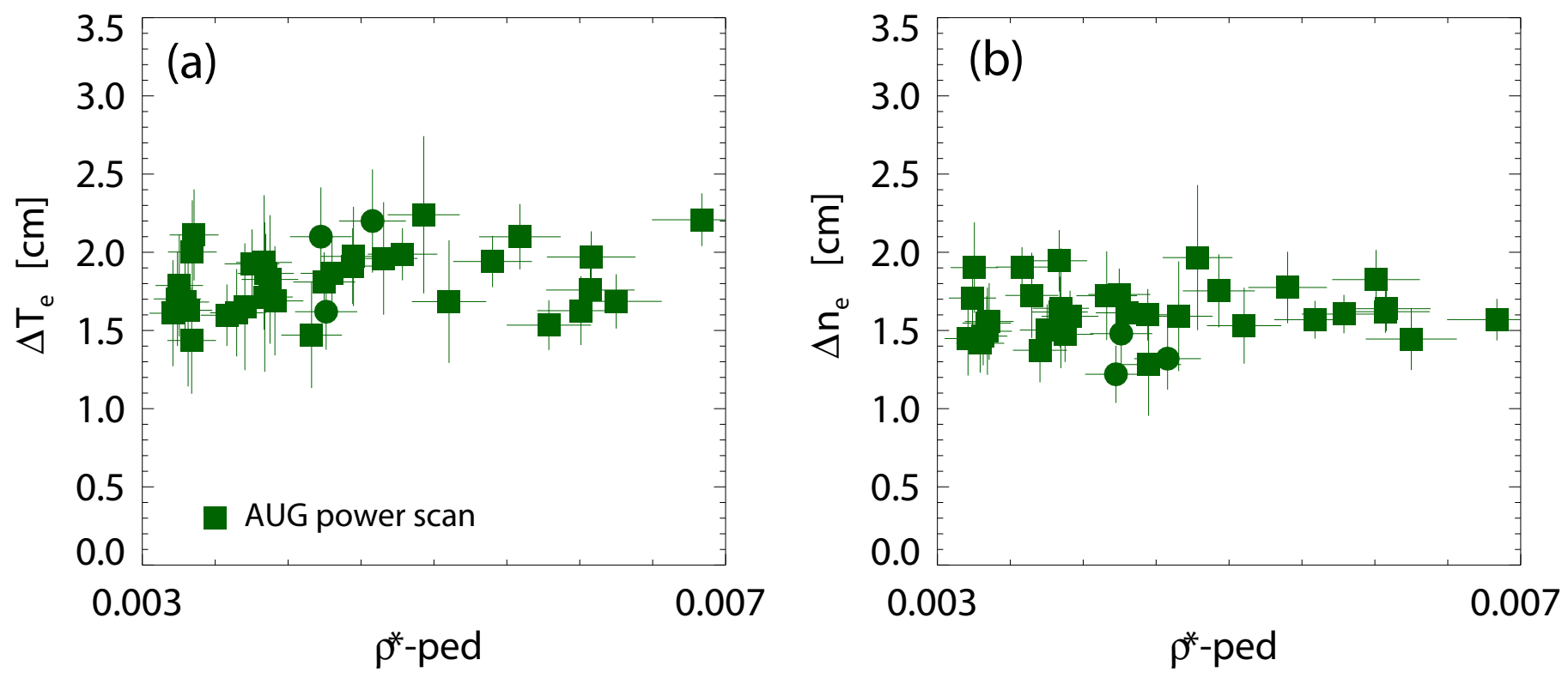



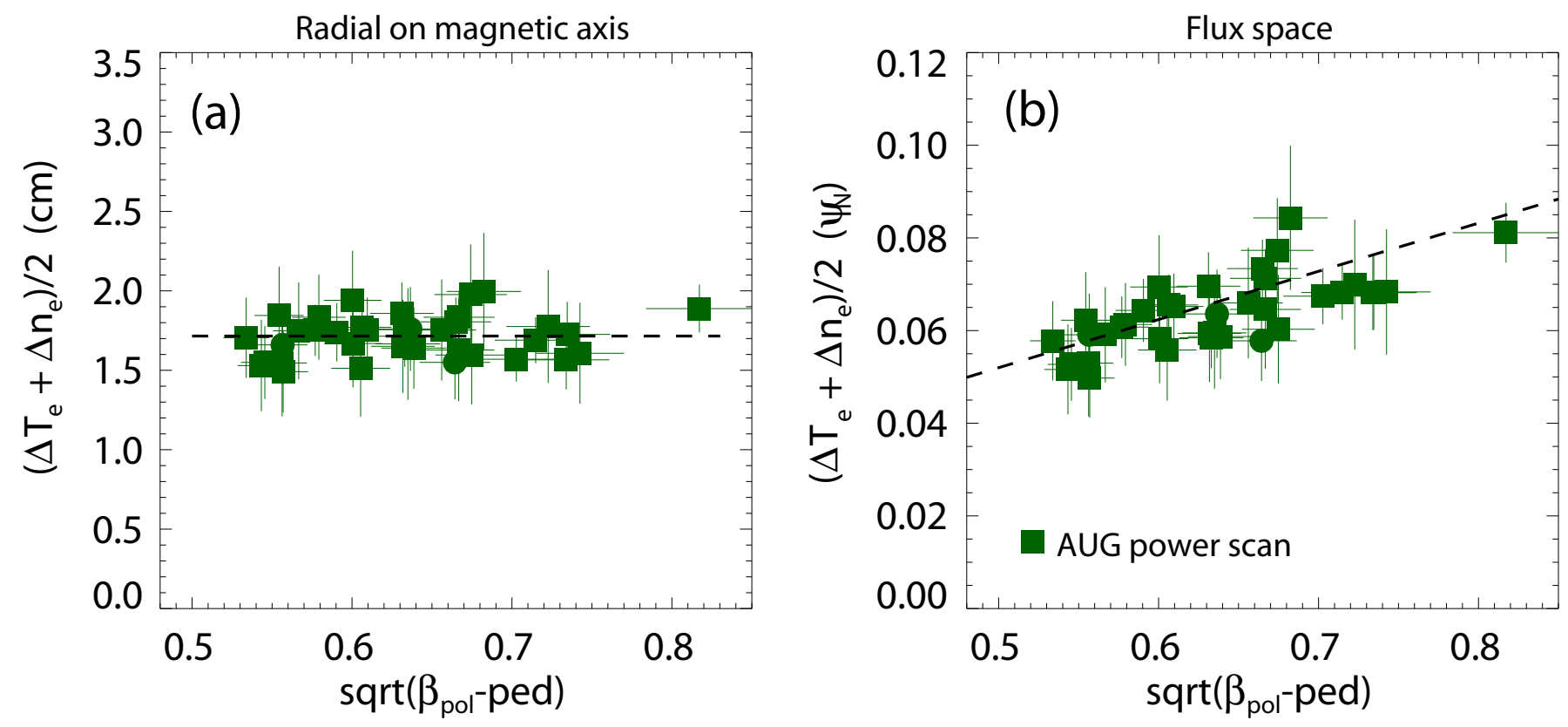

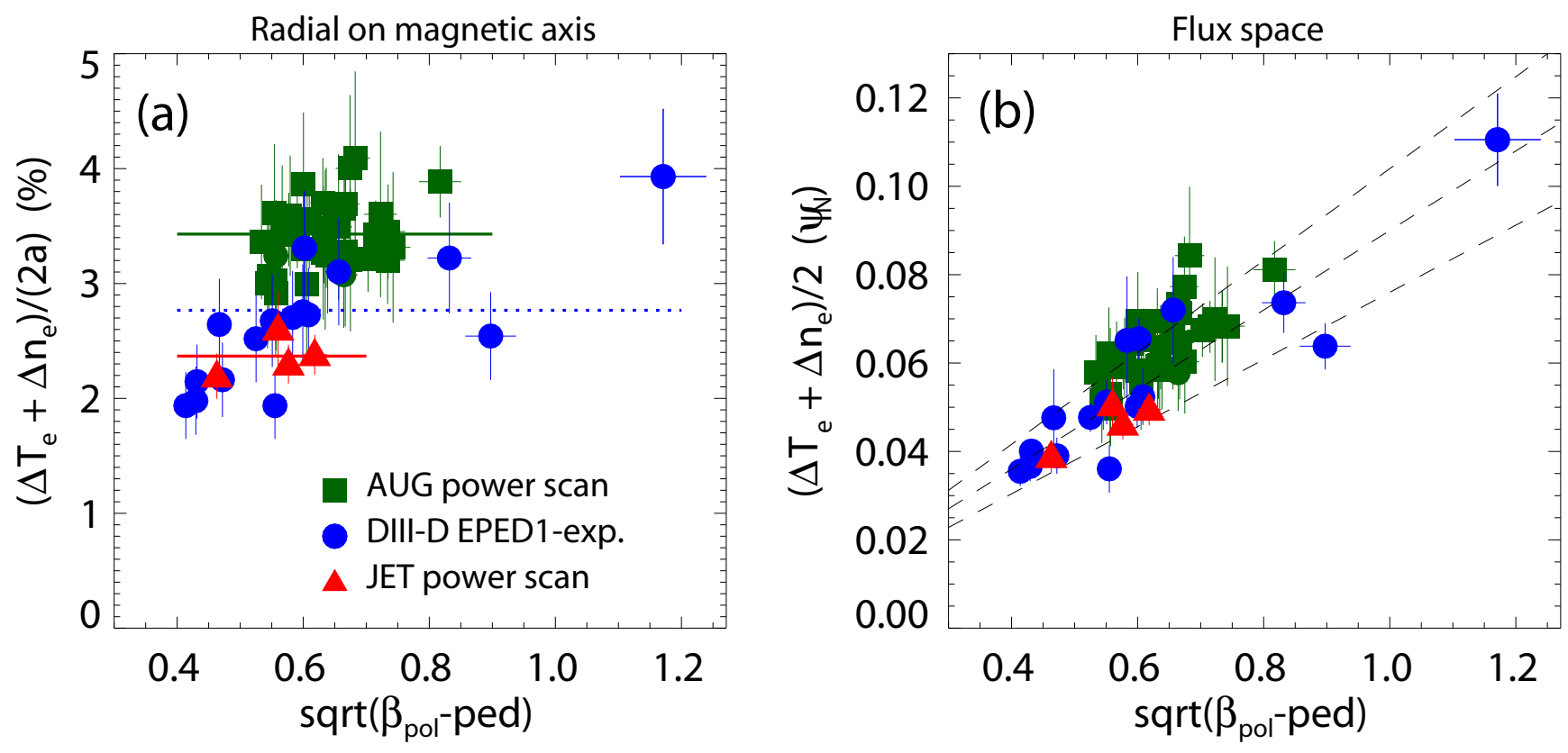


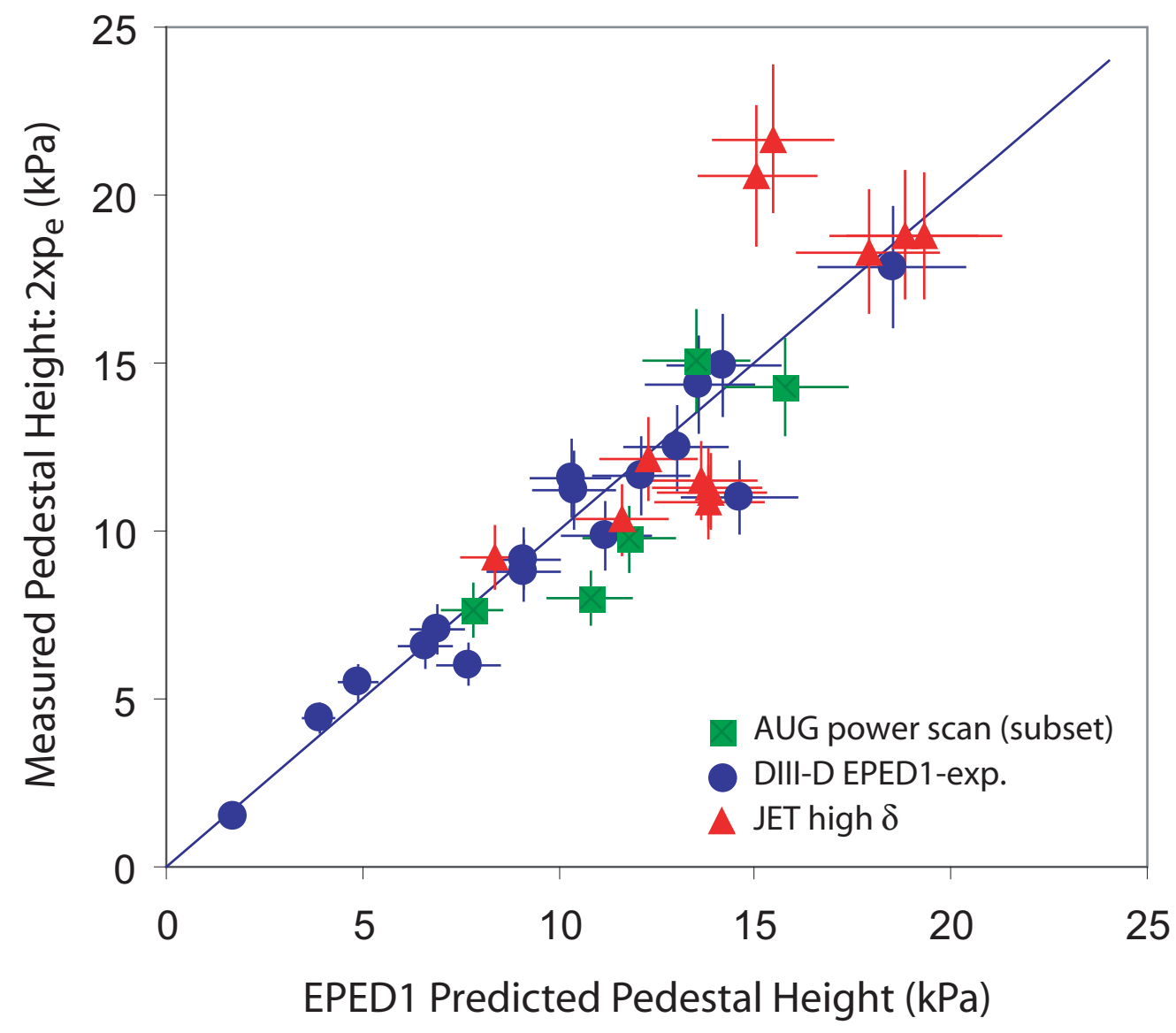

